The researchers have observed that doping an LC cell with a small amount of polyhedral oligomeric silsequioxane (POSS) nanoparticles induced spontaneous vertical alignment without the need for conven- tional alignment layers. In the July issue of Optics Letters (DOI: 10.1364/OL.33.001663; p. 1663), the researchers described the application of the NIVA technique to fabricate a GH-LCD with normally white char- acteristics and also made a plastic timepiece. The experimental display medium incorporated $5 \mathrm{wt} \%$ aminoethyl-aminopropylisobutyl-POSS nanoparticles along with $5 \mathrm{wt} \%$ dichroic dye into negative

\title{
Direct Laser Writing and CVD Combined for Fabrication of 3D Photonic Metamaterials
}

Photonic metamaterials are artificial structures with unusual properties that might lead to quantum levitation, optical cloaking, and lenses for subwavelength imaging. While natural materials at optical frequencies have a magnetic permeability $(\mu)$ of unity, metamaterials present researchers with the ability to tune $\mu$ to arbitrary values, for example, $\mu<0$. Usual building blocks of this materials class (typically periodic structures) are split ring resonators (SRRs), which effectively act as "magnetic atoms" with local magnetic dipole moments. Although most photonic metamaterials have been fabricated with two-dimensional techniques-electron-beam lithography and physical evaporation of metal filmsand stacking the functional layers, a genuinely three-dimensional (3D) fabrication process is preferable.

Toward this end, M.S. Rill and colleagues at the Institut für Angewandte Physik, Universität Karlsruhe (TH), Germany, and S. Linden and colleagues at the Institut für Nanotechnologie, Forschungszentrum Karlsruhe in der Helmholtz-Gemeinschaft, Germany, have combined direct laser writing (DLW) and silver chemical vapor deposition (CVD) to fabricate a planar test

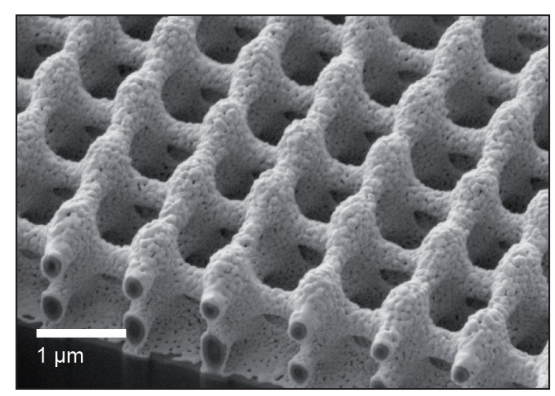

Figure 1. Oblique view of an arrangement of bars fabricated by direct laser writing and silver chemical vapor deposition after it was cut by a focused-ion beam to disclose the interior. The scanning electron micrograph (SEM) shows the potential of the presented technique to fabricate three-dimensional metallic nanostructures. Note that the silver layer covers the bars all around. structure composed of elongated SRRs. The researchers found that optical characterization of their structure is in good agreement with theory. Additionally, they fabricated a structure composed of bars, which was metallized uniformly around the structure even in 3D (see Figure 1).

As reported in the July issue of Nature Materials (DOI 10.1038/nmat2197; p. 543), the researchers made a template from a glass substrate covered with a 2- $\mu \mathrm{m}$ thick polymerized resist film (SU-8). Onto this film, a second SU-8 film was spun, exposing it using DLW, baking, and developing. A thin $\mathrm{SiO}_{2}$ coating (several dozen nanometers thick), which provides the SU- 8 backbone with mechanical stability and chemical protection, was then applied by using atomic layer deposition. CVD of Ag was then performed using the metal-organic precursor (COD)(hfac) $\mathrm{Ag}(\mathrm{I})$ at $160^{\circ} \mathrm{C}$; about $5 \mathrm{~nm}$ of $\mathrm{Ag}$ was deposited during a 40-min cycle. The structures resulted from 10 cycles for a total Ag thickness of $\sim 50 \mathrm{~nm}$.

Figure 2 shows a schematic of the structures (with geometrical parameters $a, d$, and $w$-the period, height, and width of the arrangement of SRRs, respectively) and an electron micrograph of the actual structure. The Ag coating is uniform, although somewhat granular and, importantly, has no discontinuities, which the researchers demonstrated with good dc conductivity and a reflectance greater than $95 \%$ in the wavelength range of $1-4 \mu \mathrm{m}$. Normal-incidence transmittance spectra, with incident light polarized vertically to the grooves of the elongated SRRs, showed that different heights $(d)$ correspond to different fundamental resonance frequencies.

The researchers said that the effectivemedium approximation is well justified, particularly for the structure with $a=800$ $\mathrm{nm}$ and $d=740 \mathrm{~nm}$, which shows a value of 4 for $\lambda / a$, that is, well separated from the Rayleigh anomalies and the Bragg condition. The researchers then numerically simulated the optical spectra using 3D finite-difference time-domain calculations, which agree well with experiment, and extracted from there the
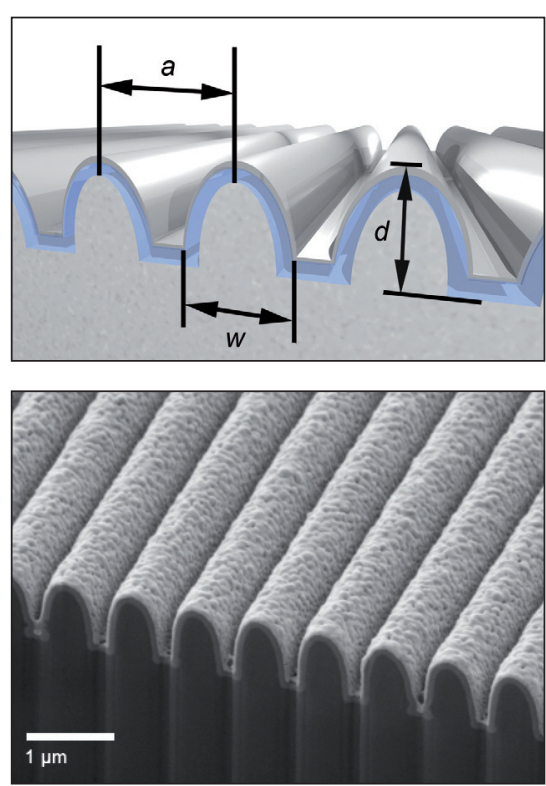

Figure 2. (a) Schematic of a planar lattice of elongated split ring resonators, all metal parts connected; polymer (light grey), silica (blue), and silver (dark grey, reflective). (b) SEM of a metamaterial corresponding to the design schematically shown in Figure $2 a$. The focused-ion beam cut reveals the $\mathrm{SiO}_{2}$ layer between the SU-8 template and the silver coating.

Reproduced with permission from Nature Materials 7 (July) DOI: 10.1038/nmat2197; p. 543. ()2008 Macmillan Publishers Ltd.

effective optical parameters-the complex permittivity, the complex permeability, and the bi-anisotropy parameter, which describes the excitation of magnetic dipoles by the electric component of the field and vice versa. For a fairly broad frequency interval around 100 $\mathrm{THz}$ ( $3 \mu \mathrm{m}$ wavelength), the real part of $\mu$ is negative, showing that the researchers have indeed fabricated a magnetic metamaterial.

The researchers said, "Theory has not yet provided blueprints for 3D metamaterials, but perhaps our approach, which enables rapid prototyping of complex 3D photonic metamaterials, will stimulate theoretical progress."

STEVEN TROHALAKI 